Hozhabr Jamali Atergeleh, Mohammad Hassan Emamian*, Shahrbanoo Goli, Marzieh Rohani-Rasaf, Hassan Hashemi and Akbar Fotouhi

\title{
The risk factors of COVID-19 in 50-74 years old people: a longitudinal population-based study
}

https://doi.org/10.1515/em-2021-0024

Received May 27, 2021; accepted December 7, 2021; published online January 10, 2022

\section{Abstract}

Objectives: To investigate the risk factors of COVID-19 infection in a longitudinal study of a population aged 50-74 years.

Methods: Data were collected from Shahroud Eye Cohort study and the COVID-19 electronic registry in Shahroud, northeast Iran. Participants were followed for about 13 months and predisposing factors for COVID-19 infection were investigated using log binominal model and calculating relative risks.

Results: From the beginning of the COVID-19 outbreak in Shahroud (February 20, 2020) to March 26, 2021, out of 4,394 participants in the Eye Cohort study, 271 (6.1\%) were diagnosed with COVID-19 with a positive reverse transcription polymerase chain reaction test on two nasopharyngeal and oropharyngeal swabs. Risk factors for COVID-19 infection included male gender (relative risk $(\mathrm{RR})=1.51 ; 95 \%$ confidence intervals (CI), 1.15-1.99), body mass index (BMI) over $25(\mathrm{RR}=1.03$; 95\% CI, 1.01-1.05), and diabetes ( $\mathrm{RR}=1.31 ; 95 \% \mathrm{CI}$, 1.02-1.67). Also, smoking ( $\mathrm{RR}=0.51 ; 95 \% \mathrm{CI}, 0.28-0.93)$ and education $(\mathrm{RR}=0.95 ; 95 \% \mathrm{CI}, 0.92-0.98)$ showed inverse associations.

Conclusions: Men, diabetics, and those with BMI over 25 should be more cognizant and adhere to health protocols related to COVID-19 prevention and should be given priority for vaccination.

Keywords: COVID-19; Iran; risk factors.

\section{Introduction}

COVID-19, which was first reported from China in 2019 and became a pandemic within a few months, is a threat to human society and has challenged all aspects of human life. As of April 5, 2021, it has infected more than 130 million people worldwide and caused more than 2.8 million deaths. In Iran, more than 1.9 million

*Corresponding author: Mohammad Hassan Emamian, MD, PhD, Ophthalmic Epidemiology Research Center, Shahroud University of Medical Sciences, Shahroud, Iran, E-mail:emamian@shmu.ac.ir. https://orcid.org/0000-0002-1994-1105

Hozhabr Jamali Atergeleh, Student Research Committee, School of Public Health, Shahroud University of Medical Sciences, Shahroud, Iran. https://orcid.org/0000-0001-7944-7937

Shahrbanoo Goli, Department of Epidemiology, School of Public Health, Shahroud University of Medical Sciences, Shahroud, Iran. https://orcid.org/0000-0003-4066-6627

Marzieh Rohani-Rasaf, Center for Health Related Social and Behavioral Sciences Research, Shahroud University of Medical Sciences, Shahroud, Iran. https://orcid.org/0000-0002-7945-7542

Hassan Hashemi, Noor Ophthalmology Research Center, Noor Eye Hospital, Tehran, Iran.

https://orcid.org/0000-0002-2109-0856

Akbar Fotouhi, Department of Epidemiology and Biostatistics, School of Public Health, Tehran University of Medical Sciences, Tehran, Iran. https://orcid.org/0000-0002-6438-6833 
cases have been identified and more than 62,000 deaths have been reported (World Health Organization 2021). One of the characteristics of this disease is its rapid transmission; therefore, it is necessary to identify ways to prevent and treat this disease to restore normal conditions. As such, it is essential to identify predisposing factors which can inform preventative and control measures.

Studies on the risk factors for COVID-19 infection have identified factors like age, sex, race, occupation, level of education, and social and economic status (Chadeau-Hyam et al. 2020; Liu et al. 2020; Pasco et al. 2020; Pijls et al. 2021; Rozenfeld et al. 2020) and underlying diseases such as cardiovascular disease, chronic kidney disease, and hypertension (Chang et al. 2020). Most of this information is obtained from crosssectional studies that may have biases related to this type of study, while longitudinal studies can provide more accurate information. Some longitudinal studies have investigated COVID-19 in Iran (Jalili et al. 2021; Javanian et al.2020), but they focused on risk factors of severe disease and death, and they had short follow-up periods. Therefore, this longitudinal study was designed to investigate risk factors of COVID-19 infection with a 13-month follow up.

\section{Materials and methods}

\section{Study population and sample size}

The sample for this study were recruited from the Shahroud Eye Cohort Study (ShECS) which has been conducted in three phases at five-year intervals in the adult population of Shahroud, in northeast Iran. ShECS began in 2009 by inviting 6,311 people aged 40-64 years oldand enrolling 5,190 respondents who entered the study (Fotouhi et al. 2013). In the first phase, people were selected by random selection of 300 clusters, each one with at least 20 participants. Many demographic factors such as gender, age, marital status, education, occupation, as well as information about smoking habits, presence of underlying diseases, blood group, body mass index (BMI), blood pressure, and certain clinical and laboratory tests are recorded as part of ShECS. The third phase of ShECS began in 2019 and completed with 4,394 participants aged 50-74 years before the COVID-19 outbreak began in Iran. In the present study, all participants of the third phase of ShECS were followed for COVID-19 infection over a 13-month period.

\section{Data and definitions}

The required data were extracted from the ShECS database for all participants. Then the COVID-19 electronic registry was searched to identify ShECS participants who had COVID-19 symptoms and were screened for COVID-19.

The definitive cases of COVID-19 were those who presented at health centers with symptoms of the disease and whose reverse transcription-polymerase chain reaction (RT-PCR) test on nasopharyngeal and oropharyngeal swabs was positive.

In categorizing data, widowers and divorcees were recoded as single. Cases with a systolic blood pressure $\geq 140 \mathrm{mmHg}$ and/or diastolic blood pressure was $\geq 90 \mathrm{mmHg}$, or those taking antihypertensive medications were categorized as hypertensive. People with diabetes were those with a fasting blood glucose $\geq 7 \mathrm{mmol} / \mathrm{L}$ and/or people taking glucose-lowering medicines. Dyslipidemia was defined as a triglyceride level $\geq 2.26 \mathrm{mmol} / \mathrm{L}$, cholesterol $\geq 6.21 \mathrm{mmol} / \mathrm{L}$, HDL-C $<1.03 \mathrm{mmol} / \mathrm{L}$, or using lipid-lowering medications.

\section{Statistical analysis}

For statistical analysis, mean and standard deviation or relative frequencies of variables were calculated in both COVID-19 positive and negative cases based on RT-PCR test results. The association between the outcome of interest (COVID-19 infection) and the studied variables were examined using independent samples t-test or chi-square test. Simple and multiple binomial regression models were used to calculate unadjusted and adjusted relative for COVID-19 infection.

\section{Ethics}

The Shahroud Eye Cohort Study and present study were approved by the ethics committee of Shahroud University of Medical Sciences. (Reference numbers: IR.SHMU.REC.1398.039, and IR.SHMU.REC.1400.016) We obtained written informed consent from all participants of ShECS after explaining the methods and purpose of the research, allowing sufficient time for questions, and ensuring clarity. 


\section{Results}

From the date of the first registered case of COVID-19 outbreak in Shahroud (February 20, 2020) to March 26, 2021, 271 of the 4,394 ShECS III participants (6.1\%) were diagnosed with COVID-19 infection using RT-PCR testing. Table 1 summarizes demographics and risk factors in the two study groups and the total sample.

The mean age of the total sample was 61.1 years, and 59.4\% of them were female. Dyslipidemia (84.4\%) and hypertension (63.4\%) were the most common underlying diseases. The most and least common blood groups were $\mathrm{O}(34.7 \%)$ and $\mathrm{AB}(7.8 \%)$, respectively.

The mean age of COVID-19 PCR-positive cases (62.0 years) was higher than that of negative cases (61.0 years). Also, the prevalence of smoking was higher among non-positive individuals. Mean BMI as well as the frequency of dyslipidemia were higher in PCR-positive cases. The mean years of education in non-positive cases were higher than PCR-positive cases.

Table 1: Demographic characteristics and past medical history of participants by polymerase chain reaction (PCR) test results, Shahroud, Iran.

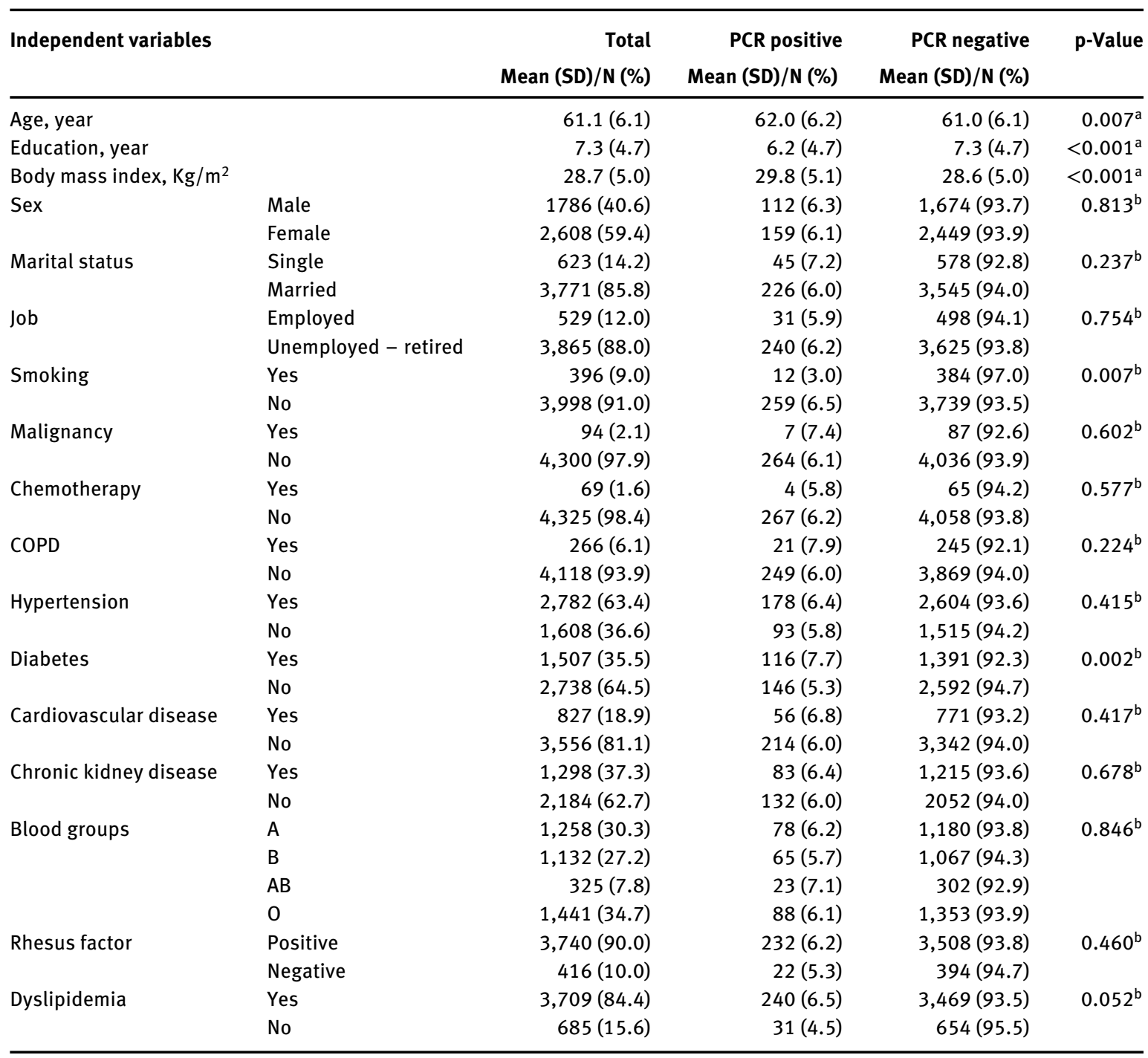

COPD, Chronic obstructive pulmonary disease; SD, standard deviation; ${ }^{a}$ independent samples t-test, ${ }^{\mathrm{b}}$ Chi-square. 
In simple binomial regression models, the relation between each independent variable and the COVID-19 was calculated. Among the studied variables, age, diabetes, smoking, BMI, and education had a significant relation with the outcome (Table 2). All variables that significantly associated with the outcome measure in simple model were entered into multiple binomial regression model. In latest model, the RR of COVID-19 for males was 1.51 (95\% CI, 1.15-1.99). There was a greater risk of COVID-19 infection with higher BMI (RR = 1.03; 95\% CI, 1.01-1.05) and diabetes ( $R R=1.31$; 95\% CI, 1.02-1.67), and the risk was lower with smoking and higher level of education $(\mathrm{RR}=0.51 ; 95 \% \mathrm{CI}, 0.28-0.93$ and $\mathrm{RR}=0.95 ; 95 \% \mathrm{CI}, 0.92-0.98$, respectively) (Table 2).

\section{Discussion}

Identifying risk factors and high-risk groups for COVID-19 are of particular importance in controlling and reducing the burden of disease in communities. In the present study, male gender, high BMI, low education, and diabetes were associated with higher risk of COVID-19 infection.

Male gender has been identified as a risk factor for severe COVID-19 morbidity and mortality in other studies (Haitao et al. 2020; Nasiri et al. 2020). In a meta-analysis of 57 studies, the prevalence of COVID-19 was higher in men (Abate et al. 2020). This difference may be attributed to the intergender difference in molecular and sex hormones, as well as lifestyle patterns and behaviors. The higher rate of employment among men (83.2\% in the present study) as well as more risky behaviors and non-compliance with health and nutrition recommendations can be reasons for this increased risk. Alcohol and tobacco use are other factors that make men more susceptible to diseases, although in the current study, smoking was a protective factor.

Table 2: The association of demographic variables and past medical conditions with COVID-19 in log binominal regression models, Shahroud, Iran.

\begin{tabular}{|c|c|c|c|c|c|c|}
\hline \multirow[t]{2}{*}{ Independent variables } & \multicolumn{3}{|c|}{ Simple } & \multicolumn{3}{|c|}{ Multiple } \\
\hline & $\mathbf{R R}$ & $95 \% \mathrm{Cl}$ & $\overline{p-V a l u e}$ & $\mathbf{R R}$ & $95 \% \mathrm{Cl}$ & $\overline{p-V a l u e}$ \\
\hline Age & 1.02 & $1.00-1.04$ & 0.007 & 1.01 & $0.99-1.03$ & 0.316 \\
\hline Male sex & 1.02 & $0.81-1.30$ & 0.813 & 1.51 & $1.15-1.99$ & 0.003 \\
\hline Being married & 0.82 & $0.60-1.12$ & 0.236 & & & \\
\hline Job & 0.94 & $0.65-1.35$ & 0.754 & & & \\
\hline Having malignancy & 1.21 & $0.58-2.49$ & 0.600 & & & \\
\hline COPD & 1.30 & $0.85-2.00$ & 0.222 & & & \\
\hline Smoking & 0.46 & $0.26-0.82$ & 0.009 & 0.51 & $0.28-0.93$ & 0.030 \\
\hline Under chemotherapy & 0.93 & $0.36-2.44$ & 0.898 & & & \\
\hline Hypertension & 1.10 & $0.86-1.41$ & 0.416 & & & \\
\hline Diabetes & 1.44 & $1.14-1.82$ & 0.002 & 1.31 & $1.02-1.67$ & 0.031 \\
\hline CVD & 1.12 & $0.84-1.49$ & 0.416 & & & \\
\hline CKD & 1.05 & $0.81-1.38$ & 0.678 & & & \\
\hline Dyslipidemia & 1.42 & $0.99-2.05$ & 0.055 & & & \\
\hline Education (year) & 0.95 & $0.92-0.97$ & $<0.001$ & 0.95 & $0.92-0.98$ & 0.002 \\
\hline BMI & 1.04 & $1.02-1.06$ & $<0.001$ & 1.03 & $1.01-1.05$ & 0.001 \\
\hline Positive Rh & 0.85 & $0.55-1.30$ & 0.462 & & & \\
\hline \multicolumn{7}{|l|}{ Blood groups } \\
\hline$A B$ (reference) & 1 & & & & & \\
\hline A & 0.87 & $0.55-1.37$ & 0.564 & & & \\
\hline B & 0.81 & $0.51-1.28$ & 0.372 & & & \\
\hline 0 & 0.86 & $0.55-1.34$ & 0.514 & & & \\
\hline
\end{tabular}

$\mathrm{Cl}$, Confidence interval; COPD, chronic obstructive pulmonary disease; CVD, cardiovascular disease; CKD: chronic kidney disease; $\mathrm{BMI}$ : body mass index $\left(\mathrm{kg} / \mathrm{m}^{2}\right)$; $\mathrm{RH}$, Rhesus ( $\left.\mathrm{Rh}\right)$ factor. 
There are other studies that have shown smoking as a protective factor for COVID-19 infection (Paleiron et al. 2021; Green et al. 2020) and even better disease prognosis (de Bernardis and Busà 2020; Kloc, Ghobrial, and Kubiak 2020; Simons et al. 2020; Tajlil et al. 2020), but other studies have indicated worse outcomes and higher COVID-19 death rates among smokers (Hamer et al. 2020; Patanavanich and Glantz 2020; Wilson 2020; Zhao et al. 2020; Zheng et al. 2020). Some have suggested the involvement of tobacco mosaic virus (TMV) in antibody production and interferon secretion in smokers as a protective mechanism against COVID-19 infection (de Bernardis and Busà 2020). Even current smokers appear to be more protected against COVID-19 than former smokers (Farsalinos et al. 2020). Given the mass media campaigns against smoking, it can be assumed that smokers may follow COVID-19 guidelines, such as social distancing and wearing a mask, because they feel more at risk for COVID-19 infection. There is also evidence that shows the desire to quit smoking and not smoking indoors has increased since the COVID-19 pandemic (Chertok 2020). Most of these studies are cross-sectional, and more powerful studies should be conducted longitudinally to examine the true effect of smoking on COVID-19 infection and outcome.

A study in India showed that a higher BMI is a risk factor for COVID-19 infection (Ranjan et al. 2020), which is in agreement with our results where each unit increase in BMI increased the risk of COVID-19 by three percent. High BMI is a risk factor for many diseases, as being overweight and obese disrupts the immune system (de Heredia, Gómez-Martínez, and Marcos 2012). Obese or overweight people may even have more occupationally high-risk behaviors or follow COVID-19 prevention protocols less carefully. Being overweight can even affect disease prognosis; studies show that a higher BMI can be a risk factor for infection and worse symptoms, as well as a predictor of intensive care unit (ICU) admission and even death (de Siqueira et al. 2020; Földi et al. 2020; Huang et al. 2020).

Diabetes was another risk factor for COVID-19 infection in this study. Other studies showed no difference in COVID-19 infection prevalence between the diabetic population and the general population (Pugliese et al. 2020), while diabetes was a predictor of ICU admission and death in infected patients (Singh et al. 2020). A weak immune system in diabetic patients puts them at greater risk for various infections (Ferlita et al. 2019; Geerlings and Hoepelman 1999), and the same can be true in case of COVID-19. COVID-19 infection in diabetic patients can even cause ketoacidosis ( $\mathrm{Li}$ et al. 2020), be more difficult to control, and increase mortality rates (Orioli et al. 2020).

Another factor that was examined in the present study and found to be a protective factor was the level of education. We found that each year increase in the level of education reduces the risk of COVID-19 by five percent. Some other studies show that a higher level of COVID-19 awareness, which is associated with the level of individuals' education, is a strong predictor of infection (Chadeau-Hyam et al. 2020; Zhong et al. 2020). Many factors can be involved in this relationship. Some examples to mention are that people with more education have a better economic status and can afford to follow stay home protocols, their better awareness due to more reading and more access to the media, and adhering to social distancing and health protocols.

High blood pressure and being employed are among studied variables that were not associated with COVID-19 infection in the current study, but they have been suggested as risk factors in other studies (Chang et al. 2020; Chadeau-Hyam et al. 2020). The reason may be the high prevalence of hypertension (Khosravi et al. 2014) and unemployment in this study sample. In this study, cancer patients did not show higher rates of COVID-19 infection, which may be due to the small sample size of this group of participants and low statistical power. Studies have shown different COVID-19 infection rates based on blood group and Rh types (Liu et al. 2021; Pourali et al. 2020; Wu et al. 2020), but this was not observed in our study. This could be due to differences in study design, participants' age range and distribution, and epidemic severity. Finally older age, which has been identified as a risk factor for COVID-19 (Rozenfeld et al. 2020), was not significant in our study, which may be due to the limited age range and high mean age in this study.

The strengths of this study include its longitudinal design and large sample size. However, it only included people aged 50-74 years, and its results may not be generalized to other age groups. The small size of certain subgroups (e.g. participants with malignancy or under chemotherapy) may have affected the results as well. 
Another limitation of this study is the lack of information about the level of knowledge and adherence to COVID-19 health protocols among the participants.

\section{Conclusions}

The results of this longitudinal study showed that being male, having a higher BMI, and being diabetic can increase the risk of COVID-19 infection among the population of 50-74 years. Also, in this age group, having higher education has a protective role against this disease. The lower risk of COVID-19 among smokers needs to be examined more closely to determine whether this is related to smoking or their behavioral patterns. High risk groups should be informed more about COVID-19, and it is recommended that they be given priority in vaccination programs.

Acknowledgments: Not applicable.

Research funding: The Shahroud eye cohort study was supported by the Noor Ophthalmology Research Center and Shahroud University of Medical Sciences (Grant Numbers: 9826 and 99142).

Author contribution: Conceptualization: MHE, HH, AF. Data curation: MHE, MRR, SG, HJA. Formal analysis: HJA, MHE, SG, MRR. Funding acquisition: HH. Methodology: MHE, SG. Writing - original draft: HJA. Writing - review \& editing: MHE, AF, HH, SG, MRR. All authors have accepted responsibility for the entire content of this manuscript and approved its submission.

Competing interests: Authors state no conflict of interest.

Informed consent: Written informed consent was obtained from all individuals included in this study.

Ethical approval: The local Institutional Review Board at Shahroud University of Medical Sciences approved this study.

\section{References}

Abate, B. B., A. M. Kassie, M. W. Kassaw, T. G. Aragie, and S. A. Masresha. 2020. "Sex Difference in Coronavirus Disease (COVID-19): A Systematic Review and Meta-Analysis.” BMJ Open 10 (10): e040129.

Chadeau-Hyam, M., B. Bodinier, J. Elliott, M. D. Whitaker, I. Tzoulaki, R. Vermeulen, M. Kelly-Irving, C. Delpierre, and P. Elliott. 2020. "Risk Factors for Positive and Negative COVID-19 Tests: A Cautious and In-Depth Analysis of UK Biobank Data." International Journal of Epidemiology 49 (5): 1454-67.

Chang, T. S., Y. Ding, M. K. Freund, R. Johnson, T. Schwarz, J. M. Yabu, C. Hazlett, J. N. Chiang, A. Wulf, UCLA Health Data Mart Working Group, D. H. Geschwind, M. J. Butte, and B. Pasaniuc. 2020. "Prior Diagnoses and Medications as Risk Factors for COVID-19 in a Los Angeles Health System.” medRxiv. https://doi.org/10.1101/2020.07.03.20145581.

Chertok, I. R. A. 2020. "Perceived Risk of Infection and Smoking Behavior Change during COVID-19 in Ohio." Public Health Nursing 37 (6): 854-62.

de Bernardis, E., and L. Busà. 2020. "A Putative Role for the Tobacco Mosaic Virus in Smokers' Resistance to COVID-19." Medical Hypotheses 143: 110153.

de Heredia, F. P., S. Gómez-Martínez, and A. Marcos. 2012. "Obesity, Inflammation and the Immune System." Proceedings of the Nutrition Society 71 (2): 332-8.

de Siqueira, J. V. V., L. G. Almeida, B. O. Zica, I. B. Brum, A. Barceló, and A. G. de Siqueira Galil. 2020. “Impact of Obesity on Hospitalizations and Mortality, Due to COVID-19: A Systematic Review.” Obesity Research \& Clinical Practice 14 (5): $398-403$.

Farsalinos, K., A. Barbouni, K. Poulas, R. Polosa, P. Caponnetto, and R. Niaura. 2020. “Current Smoking, Former Smoking, and Adverse Outcome Among Hospitalized COVID-19 Patients: A Systematic Review and Meta-Analysis.” Ther Adv Chronic Dis. 11: 2040622320935765.

Ferlita, S., A. Yegiazaryan, N. Noori, G. Lal, T. Nguyen, K. To, and V. Venketaraman. 2019. "Type 2 Diabetes Mellitus and Altered Immune System Leading to Susceptibility to Pathogens, Especially Mycobacterium tuberculosis.” Journal of Clinical Medicine 8 (12): 2219.

Földi, M., N. Farkas, S. Kiss, N. Zádori, S. Váncsa, L. Szakó, F. Dembrovszky, M. Solymár, E. Bartalis, Z. Szakács, P. Hartmann, G. Pár, B. Eróss, Z. Molnár, P. Hegyi, and A. Szentesi. 2020. "Obesity is a Risk Factor for Developing Critical Condition in COVID-19 Patients: A Systematic Review and Meta-Analysis.” Obesity Reviews 21 (10): e13095. 
Fotouhi, A., H. Hashemi, M. Shariati, M. H. Emamian, K. Yazdani, E. Jafarzadehpur, H. Koohian, M. R. Khademi, K. Hodjatjalali, A. Kheirkhah, R. Chaman, S. Malihi, M. Mirzaii, and M. Khabazkhoob. 2013. "Cohort Profile: Shahroud Eye Cohort Study." International Journal of Epidemiology 42 (5): 1300-8.

Geerlings, S. E., and A. I. M. Hoepelman. 1999. "Immune Dysfunction in Patients with Diabetes Mellitus (DM)." FEMS Immunology and Medical Microbiology 26 (3-4): 259-65.

Green, I., E. Merzon, S. Vinker, A. Golan-Cohen, and E. Magen. 2021. “COVID-19 Susceptibility in Bronchial Asthma.” J Allergy Clin Immunol Pract. 9 (2): 684-92.e1.

Haitao, T., J. V. Vermunt, J. Abeykoon, R. Ghamrawi, M. Gunaratne, M. Jayachandran, K. Narang, S. Parashuram, S. Suvakov, and V. D. Garovic. 2020. “COVID-19 and Sex Differences: Mechanisms and Biomarkers.” Mayo Clinic Proceedings 95 (10): $2189-203$.

Hamer, M., M. Kivimäki, C. R. Gale, and G. D. Batty. 2020. "Lifestyle Risk Factors, Inflammatory Mechanisms, and COVID-19 Hospitalization: A Community-Based Cohort Study of 387,109 Adults in UK.” Brain, Behavior, and Immunity 87: 184-7.

Huang, Y., Y. Lu, Y. M. Huang, M. Wang, W. Ling, Y. Sui, and H. L. Zhao. 2020. “Obesity in Patients with COVID-19: A Systematic Review and Meta-Analysis." Metabolism 113: 154378.

Jalili, M., P. Payandemehr, A. Saghaei, H. N. Sari, H. Safikhani, and P. Kolivand. 2021. "Characteristics and Mortality of Hospitalized Patients with COVID-19 in Iran: A National Retrospective Cohort Study." Annals of Internal Medicine 174 (1): $125-7$.

Javanian, M., M. Bayani, M. Shokri, M. Sadeghi-Haddad-Zavareh, A. Babazadeh, B. Yeganeh, S. Mohseni, R. Mehraein, M. Sepidarkish, and A. Bijani. 2020. "Clinical and Laboratory Findings from Patients with COVID-19 Pneumonia in Babol North of Iran: A Retrospective Cohort Study.” Romanian Journal of Internal Medicine 58 (3): 161-7. (ahead-of-print).

Khosravi, A., M. H. Emamian, M. Shariati, H. Hashemi, and A. Fotouhi. 2014. "The Prevalence of Pre-hypertension and Hypertension in an Iranian Urban Population." High Blood Pressure and Cardiovascular Prevention 21 (2): 127-35.

Kloc, M., R. M. Ghobrial, and J. Z. Kubiak. 2020. "How Nicotine Can Inhibit Cytokine Storm in the Lungs and Prevent or Lessen the Severity of COVID-19 Infection?” Immunology Letters 224: 28-9.

Li, J., X. Wang, J. Chen, X. Zuo, H. Zhang, and A. Deng. 2020. "COVID-19 Infection May Cause Ketosis and Ketoacidosis." Diabetes, Obesity and Metabolism 22 (10): 1935-41.

Liu, N., T. Zhang, L. Ma, H. Zhang, H. Wang, W. Wei, H. Pei, and H. LI. 2021. "The Impact of ABO Blood Group on COVID-19 Infection Risk and Mortality: A Systematic Review and Meta-Analysis.” Blood Rev 48: 100785.

Liu, R., H. Han, F. Liu, Z. Lv, K. Wu, Y. Liu, Y. Feng, and C. Zhu. 2020. "Positive Rate of RT-PCR Detection of SARS-CoV-2 Infection in 4880 Cases from One Hospital in Wuhan, China, from Jan to Feb 2020." Clinica Chimica Acta 505: 172-5.

Nasiri, M. J., S. Haddadi, A. Tahvildari, Y. Farsi, M. Arbabi, S. Hasanzadeh, P. Jamshidi, M. Murthi, and M. Mirsaeidi. 2020. "COVID-19 Clinical Characteristics, and Sex-specific Risk of Mortality: Systematic Review and Meta-Analysis." Frontiers of Medicine 7: 459.

Orioli, L., M. P. Hermans, J. P. Thissen, D. Maiter, B. Vandeleene, and J. C. Yombi. 2020. "COVID-19 in Diabetic Patients: Related Risks and Specifics of Management." Annales d'Endocrinologie 81: 101-9.

Paleiron, N., A. Mayet, V. Marbac, A. Perisse, H. Barazzutti, F.-X. Brocq, F. Janvier, D. Bertrand, and O. Bylicki. 2021. “Impact of Tobacco Smoking on the Risk of COVID-19. A Large Scale Retrospective Cohort Study." Nicotine \& Tobacco Research 23 (8): $1398-1404$.

Pasco, R. F., S. J. Fox, S. C. Johnston, M. Pignone, and L. A. Meyers. 2020. "Estimated Association of Construction Work with Risks of COVID-19 Infection and Hospitalization in Texas.” JAMA Network Open 3 (10): e2026373.

Patanavanich, R., and S. A. Glantz. 2020. "Smoking is Associated with COVID-19 Progression: A Meta-Analysis." Nicotine \& Tobacco Research 22 (9): 1653-6.

Pijls, B. G., S. Jolani, A. Atherley, R. T. Derckx, J. I. R. Dijkstra, H. L. GregorFranssen, S. Hendriks, A. Richters, A. Venemans-Jellema, and S. Zalpuri. 2021. "Demographic Risk Factors for COVID-19 Infection, Severity, ICU Admission and Death: A Meta-Analysis of 59 Studies.” BMJ open 11 (1): e044640.

Pourali, F., M. Afshari, R. Alizadeh-Navaei, J. Javidnia, M. Moosazadeh, and A. Hessami. 2020. "Relationship between Blood Group and Risk of Infection and Death in COVID-19: A Live Meta-Analysis.” New Microbes and New Infections 37: 100743.

Pugliese, G., M. Vitale, V. Resi, and E. Orsi. 2020. “Is Diabetes Mellitus a Risk Factor for COronaVIrus Disease 19 (COVID-19)?” Acta Diabetologica 57 (11): 1275-1285.

Ranjan, P., A. Kumar, S. Chowdhury, S. Pandey, A. Choudhary, A. Bhattacharya, A. Singh, R. M. Pandey, N. Wig, and N. K. Vikram. 2020. "Is Excess Weight a Risk Factor for the Development of COVID 19 Infection? A Preliminary Report from India.” Diabetes \& Metabolic Syndrome: Clinical Research Reviews 14 (6): $1805-7$.

Rozenfeld, Y., J. Beam, H. Maier, W. Haggerson, K. Boudreau, J. Carlson, and R. Medows. 2020. “A Model of Disparities: Risk Factors Associated with COVID-19 Infection." International Journal for Equity in Health 19 (1): 1-10.

Simons, D., L. Shahab, J. Brown, and O. Perski. 2020. "The Association of Smoking Status with SARS-CoV-2 Infection, Hospitalization and Mortality from COVID-19: A Living Rapid Evidence Review with Bayesian Meta-analyses (Version 7)." Addiction 116 (6): 1319-68. 
Singh, A. K., R. Gupta, A. Ghosh, and A. Misra. 2020. "Diabetes in COVID-19: Prevalence, Pathophysiology, Prognosis and Practical Considerations.” Diabetes \& Metabolic Syndrome: Clinical Research Reviews 14 (4): 303-10.

Tajlil, A., S. Ghaffari, L. Pourafkari, S. Mashayekhi, and N. Roshanravan. 2020. "Nicotine and Smoking in the COVID-19 Era." Journal of Cardiovascular and Thoracic Research 12 (2): 136-9.

Wilson, C. 2020. "Smokers Are Actually at a Higher Risk of Dying from Covid-19." New Scientist 246 (3283): 8-9.

World Health Organization. 2021. WHO Coronavirus (COVID-19) Dashboard. https://covid19.who.int/ (accessed May 4, 2021).

Wu, B.-B., D.-Z. Gu, J.-N. Yu, J. Yang, and W.-Q. Shen. 2020. "Association between ABO Blood Groups and COVID-19 Infection, Severity and Demise: A Systematic Review and Meta-Analysis." Infection, Genetics and Evolution 84: 104485.

Zhao, Q., M. Meng, R. Kumar, Y. Wu, J. Huang, N. Lian, Y. Deng, and S. Lin. 2020. "The Impact of COPD and Smoking History on the Severity of COVID-19: A Systemic Review and Meta-Analysis.” Journal of Medical Virology 92 (10): $1915-21$.

Zheng, Z., F. Peng, B. Xu, J. Zhao, H. Liu, J. Peng, Q. Li, C. Jiang, Y. Zhou, S. Liu, C. Ye, P. Zhang, Y. Xing, H. Guo, and W. Tang. 2020. "Risk Factors of Critical \& Mortal COVID-19 Cases: A Systematic Literature Review and Meta-Analysis." Journal of Infection 81 (2): e16-e25.

Zhong, B.-L., W. Luo, H.-M. Li, Q.-Q. Zhang, X.-Ge. Liu, W.-T. Li, and Yi. Li. 2020. “Knowledge, Attitudes, and Practices towards COVID-19 Among Chinese Residents during the Rapid Rise Period of the COVID-19 Outbreak: A Quick Online Cross-Sectional Survey." International Journal of Biological Sciences 16 (10): 1745-52. 